\title{
INFRAESTRUCTURA Y LOGÍSTICA EN LA HISTORIA ECONÓMICA: UNA CONTRIBUCIÓN A PARTIR DE LOS CASOS DE CHILE Y MÉXICO, CA. 1850-1970*
}

\section{INFRASTRUCTURE AND LOGISTICS IN ECONOMIC HISTORY: A CONTRIBUTION BASED ON THE CASES OF CHILE AND MEXICO, C. 1850-1970}

\author{
Guillermo Guajardo Soto \\ Universidad Nacional Autónoma de México, ciudad de México, México, \\ guillermo.guajardo@unam.mx
}

\begin{abstract}
Resumen. Se presenta un artículo sobre las relaciones entre infraestructuras y sistemas sociotécnicos del transporte en los siglos XIX y XX, para comprender las bases materiales del actual proceso de globalización y la relevancia que tiene la historia de los procesos logísticos y de las cadenas de bienes. Si bien en la historia económica se le ha dado una atención mayor a la producción de materias primas exportables y bienes industriales, en este texto analizaremos en los casos de Chile y México, entre 1850 y 1970, la movilidad de factores, su distribución y la coordinación de equipos, normas, rutinas y personal que conformaron una cadena de transformación que integró desde las materias primas al bien final. Se plantea que las infraestructuras establecieron un patrón fijo y perdurable de flujos territoriales, de larga duración, de las llamadas cadenas de movimiento.
\end{abstract}

Palabras clave: infraestructura; movilidad; ferrocarriles; capitalismo; globalización.

Abstract. This article discusses the relations between transportation infrastructures and socio-technical systems in the 19th and 20th centuries to understand the material foundations of the current globalization process and the relevance of the history of logistic processes and chains of goods. While economic history has given more importance to the production of exportable raw materials and industrial goods, in this paper, we will look at the cases of Chile and Mexico, between 1850 and 1970, and analyze the mobility of factors, their distribution and the coordination of equipment, regulations, routines, and personnel that constituted a processing chain encompassing from the raw materials to the final goods. We suggest that these infrastructures determined a fixed and enduring pattern of long-lasting territorial flows for the socalled movement chains.

\footnotetext{
* Este artículo recoge temas del proyecto de investigación Tecnologías, Organizaciones e Infraestructuras para la Innovación y la Movilidad: Un Enfoque Histórico Interdisciplinario como parte del Programa de Investigación Ciencia y Tecnología del Centro de Investigaciones Interdisciplinarias en Ciencias y Humanidades de la Universidad Nacional Autónoma de México.
} 
Key words: infrastructure; mobility; railroads; capitalism; globalization.

Fecha de recepción: 21 de marzo de 2014. Fecha de aceptación: 16 de junio de 2014.

\section{INTRODUCCIÓN: LA HISTORICIDAD DE LAS INFRAESTRUCTURAS Y FLUJOS}

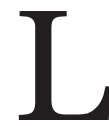

os flujos y la distribución de bienes y personas son el sello del mundo contemporáneo, resultado de un proceso histórico de interconexión de infraestructuras, tecnologías y flujos que, en su conjunto, han conformado cadenas de movilidad y de distribución cada vez más integradas. Las primeras cadenas estructuraron el comercio en la Europa medieval, y con la revolución industrial, desde la década de 1830, se difundió un paquete de coordinación de transportes e información a través del ferrocarril, los telégrafos y una red de instalaciones de manipulación y despacho de cargas. Esto hizo posible la primera globalización, así como la producción de bienes y el consumo masivo de servicios. Las cadenas continuaron durante el periodo fordista con la carretera, el camión y el avión, y tras el fin de la guerra fría se experimenta una revolución global de la logística (Braudel, 1984; Gereffi, Korzeniewicz y Korzeniewicz, 1994).

Los países de América Latina también fueron parte de las cadenas (¿de movimiento?) y su historia económica en gran parte ha estado moldeada por las infraestructuras y sistemas sociotécnicos de transporte para sus economías de exportación y mercados internos. Estos fenómenos son los que se presentan en este artículo sobre la importancia de las cadenas de materias primas, las infraestructuras y la logística en Chile y México entre 1850 y 1970. En los dos países ocurrió lo que se denomina "revoluciones de infraestructuras", que permitieron la vinculación al flujo internacional de materias primas por medio de ferrocarriles, vías, barcos y puertos, desde el siglo XIX, y en el siglo XX conformaron las bases distributivas de la industrialización, punto en el cual se cierra este análisis. Esas revoluciones -ferroviaria y carretera- hicieron posible los flujos y determinaron el proceso de path dependence sobre el territorio, fenómeno similar a otros casos, como el de Suecia estudiado por Berger y Enflo (2013), pero en América Latina, a diferencia de Escandinavia, no detonó ni promovió el desarrollo económico ni una temprana y dinámica urbanización. De ahí que estudiar los patrones de trayectoria y divergencia de una misma tecnología en distintos contextos y condiciones de arranque es una promisoria veta de análisis histórico. 


\section{Sin MOVIMIENTO NO HAY MERCADO: ¿BIENES O CADENAS EN LA CONFORMACIÓN DEL CAPITALISMO?}

Desde el siglo XIX, el movimiento y las instalaciones fijas han sido la correa de transmisión del comercio global y de la producción a través de una "coreografía tecnológica" de sacos, barcos, trenes, camiones, aviones y contenedores manipulados, almacenados y despachados desde puertos, aeropuertos, carreteras, canales, vías y patios. Si bien todos estos medios surgieron de forma aislada, hoy en día constituyen un solo compositum de industrias y servicios que movilizan, transforman y entregan bienes (Hesse, 2008). Su historia es también la construcción del mundo como una sola unidad integrada y convergente, que Baudrillard (2010) llamó en 1968 un "orden global de producción y de consumo" (pp. 4, 5 y 9). Inmanuel Wallerstein (2010) explicó esa construcción con su noción de "capitalismo histórico", que arrancó en la Europa occidental del siglo XV, para cubrir a fines del siglo XIX gran parte del globo y que hoy continúa mediante la autoexpansión del capital y la mercantilización de todos los procesos. El ciclo del capital de trabajo, mercancía, comercialización, distribución y ganancia genera procesos transnacionales, los cuales caracterizan el mundo capitalista (pp. 2-4, 12-22).

Sin embargo, O'Rourke y Williamson $(1999,2000,2004)$ difieren de Wallerstein al señalar que antes de 1800 el mundo estaba mal integrado y que en realidad el arranque de la globalización se dio en la primera parte del siglo XIX al conformarse un mercado internacional de materias primas por el declive en los costos de transporte y una convergencia de precios. Pero esto ignora que tanto Wallerstein (2010) como Gereffi, Korzeniewicz y Korzeniewicz (1994) indicaron que el movimiento de bienes se había estructurado siglos antes a través de las "cadenas de bienes", es decir, un conjunto de redes organizadas en torno a la producción de un bien determinado, que vincularon empresas y Estados con la economía mundial. Y esto implicó mejoras en la infraestructura y en la distribución, razón por la cual para Jacks, Meissner y Novy (2010) la caída en los costos del comercio internacional entre 1870 y 1913 fue no sólo por el transporte, sino por las mejoras en la infraestructura, las políticas y los avances en productividad. O'Rourke y Williamson (2004), al buscar un origen monocausal de la globalización, separaron del movimiento de factores el cambio tecnológico y el efecto geográfico de la expansión de las redes de transportes e infraestructuras y con ello su relación con el crecimiento (Harley, 2000).

En cambio, una aproximación que incluya las cadenas de bienes también integra el proceso de producción y de servicios, con lo cual se puede postular que previo a la producción de bienes han sido más relevantes las condiciones físicas para la movilidad de factores y su distribución, deto- 
nando una interacción entre lo fijo y lo fluido. En el capitalismo lo esencial no es la producción de bienes per se, sino la movilidad de factores, proceso que está en el corazón de la ganancia; sin movimiento no hay mercado. El inicio de la globalización puede entenderse a partir del comercio de bienes apilables, que escaló históricamente hacia una acumulación de procesos de interconexión en donde se desarrolló un tipo específico de movimiento económico: la coordinación de varios tipos de unidades para producir bienes o servicios para el cliente.

\section{CAdENAS DE BIENES, LOGÍsTICA Y MOVIMIENTO ECONÓMICO}

El interés por la historia de los movimientos transnacionales ya se encuentra en Braudel (1984), pero un abordaje más contemporáneo que nos interesa destacar es la historia que toma el concepto de "movilidad", introducido en la década de 1920 en las ciencias sociales por el sociólogo P. Sorokin para definir de manera unificada el cambio y el movimiento (Kaufmann, 2010, pp. 42-43). La historia de la movilidad en los últimos años ha generado un gran entusiasmo por el estudio de las personas, ideas, objetos, movimientos y fuerzas que cruzan las fronteras nacionales, así como las estructuras que los apoyan (Van der Vleuten, 2008). En todo caso ello no puede desconocer que cada país fija un marco de arranque para los recursos y tecnologías; al respecto hay bastante evidencia histórica sobre los estilos tecnológicos nacionales, como los sistemas de energía eléctrica en Estados Unidos, Alemania e Inglaterra. También en América Latina hay documentados casos de adopción y mestizaje de modelos tecnológicos internacionales antes de 1914 (Guajardo, 2001, 2005).

Tres rutas se considerarán para entender históricamente las bases materiales del movimiento de bienes. La primera es la noción de "cadena de movimiento" que integra la disponibilidad de materias primas, el transporte, la transformación y la logística. La segunda es conciliar las dimensiones nacional y transnacional mediante un estudio comparativo. Una tercera es ensamblar las rutas anteriores con los sistemas sociotécnicos que "fabrican” el movimiento en economías y sociedades.

Una tecnología e infraestructura revolucionaria que queremos considerar en nuestro análisis es el ferrocarril, aunque no de una forma reduccionista, ya que el ferrocarril durante su periodo de vigencia no fue un sistema único de transporte que superó, desplazó y desconectó otros medios. Esa imagen proviene en gran parte del estudio de Fogel (1964) sobre los ahorros sociales que introdujo el ferrocarril por la baja en los costos de transporte en la economía de Estados Unidos, donde lo caracterizó como un medio de transporte homogéneo y autosuficiente, cuya alta intensidad 
de capital desplazó a las mulas, carretas y canales sin necesitar de otros medios ni de infraestructuras. Esto permitió tanto a Fogel como a Fishlow (1965) cuestionar su impacto económico al señalar que el ingreso nacional en Estados Unidos se habría reducido sólo unos pocos puntos porcentuales en 1860 y 1890 si no hubieran existido los ferrocarriles y si el transporte se hubiera transferido a ríos, canales y otros medios.

Este enfoque ha sido poco útil para una historia de la movilidad, ya que deja de lado los procesos reales de transporte y los vínculos con otros medios que lo abastecían y que estaban presentes en los patios de carga de las estaciones ferroviarias. Las críticas (Berger y Enflo, 2013; Bogart, 2009, 2013) a este abordaje, que compartimos, pueden ser agrupadas en cinco aspectos. Primero, no quedó claro cuál habría sido el precio del transporte por carretera o canales en ausencia de los ferrocarriles. Si el ferrocarril no hubiese existido, la afluencia habría incrementado en las carreteras, canales y ríos por el crecimiento del volumen de tráfico, al tiempo que se subestimaría el costo del uso de otros modos de transporte. Segundo, el cálculo de los ahorros sociales omite los impactos económicos hacia atrás y hacia adelante por la mayor demanda de hierro y acero como insumo y el desarrollo de industrias importantes. Tercero, se dieron cambios muy relevantes en la geografía económica, ya que la reducción de los costes de transporte ocasionó aglomeración de la actividad económica y el surgimiento de las ciudades (Berger y Enflo, 2013; Bogart, 2009, 2013). Cuarto, lo más relevante es que hubo un aumento de la velocidad sobre las formas alternativas de transporte, algo central en el mundo moderno en donde "el tiempo es dinero"; el análisis de Bogart (2013) indica que el valor del tiempo ahorrado en los ferrocarriles británicos en 1870 fue por arriba de 5\% del ingreso nacional; sin la tercera clase, la mayoría de la población habría tenido que caminar al trabajo y otros destinos, y como las velocidades de a pie son de sólo 2.5 millas por hora, el cambio a una velocidad de 20 o 25 millas por hora fue fundamental.

Un quinto aspecto se refiere a la afirmación de que el desarrollo económico nacional estadunidense pudo haber tenido el mismo éxito con los canales y sin los ferrocarriles; lo anterior fue duramente criticado en la década de 1990 por John R. Meyer (1997), profesor de transporte, logística y distribución en Harvard y vicepresidente de la Union Pacific Corporation. Meyer señaló que la hipótesis de Fogel y otros era interesante intelectualmente pero irrelevante, ya que los ferrocarriles llegaron y el desarrollo económico de Estados Unidos se benefició de una sobreoferta tanto de vías fluviales como ferroviarias.

Un enfoque más comprensivo es considerar al ferrocarril como una interfaz logística conformada por su infraestructura, tecnología y organización. La máquina de vapor para uso comercial desde el decenio de 
1830 replanteó la mecánica, pero también la ingeniería civil porque se debieron construir nuevas infraestructuras e instalaciones. No podemos dejar de lado el planteamiento de Wallerstein (2010) y Gerefi y Korzeniewicz (1994), porque las cadenas globales ya existían, pero sí se debe destacar que el ferrocarril las redefinió debido a que introdujo una nueva coordinación ajustada al ciclo de negocios, gracias a la telegrafía y posteriormente a los cables submarinos que permitieron establecer redes globales de comunicación, cambiando dramáticamente la estructura de los flujos de información entre 1851 y 1902. Se desarrolló una red de inteligencia de negocios con datos sobre cotizaciones de precios e información sobre el zarpado o la llegada de barcos (Wenzlhuemer, 2010), en sintonía con los transportes marítimos y terrestres, creando las bases de la planeación y monitoreo de los flujos de bienes e información para el proceso de negocios, que hoy en día ubica a la logística como el proceso central del movimiento de bienes. ${ }^{1}$

La logística como disciplina tiene su origen en el sector militar; esta es definida como la capacidad de manejo y cálculo de los abastecimientos de todo tipo, que después de la segunda guerra mundial migró hacia los negocios. Fue en la década de 1960 cuando el experto del management estratégico, Peter F. Drucker, señaló que la logística era un área inexplorada para la ciencia económica, "un continente negro" (Allen, 1997, p. 109), porque la distribución física se consideraba como la última frontera de las economías de costos, pero emergía como una nueva frontera en la generación de la demanda. Esta falta de interés quedó reflejada en la nueva historia económica de Estados Unidos, que le restó importancia al impacto de los ferrocarriles, y con ello al transporte y a la distribución económica. Desde 1990, con la integración de Europa, la Internet y el desplazamiento de la producción de bienes a Asia, se produjo una revolución en la logística que no cesa (Vahrenkamp, 2012). Esta revolución logística se refleja en las preocupaciones de un sector de historiadores (Anastasiadou, 2009; Mom, Divall y Lyth, 2009), fundamentalmente europeos, sobre la movilidad y el transporte preocupados por los tráficos transnacionales. No obstante, la llamada de Drucker todavía sigue vigente, porque no hay una preocupación extendida en la historia económica, de la tecnología y de la empresa por esos fenómenos. Y puede aportar otra historia del ferrocarril, abordándolo como un sistema sociotécnico de movilidad ligado a su infraestructura física y subestructura normativa, para explicar cómo se con-

${ }^{1}$ Hoy en día tanto las empresas de mensajería como de ferrocarriles se definen como empresas de logística. DHL Logbook in cooperation with Technical University Darmstadt, Alemania. Recuperado de http://www.dhl-discoverlogistics.com/ 
formaron las economías y territorios de muchos países latinoamericanos, aspecto se tratará en los siguientes apartados.

\section{SISTEMAS SOCIOTÉCNICOS DE MOVILIDAD Y DISTRIBUCIÓN}

Las industrias del automóvil, aviación y aeroespacial, químicas y farmacéuticas son entidades sociotécnicas que se articulan en torno a una tecnología que define a un sector, y que moldean patrones específicos de organización industrial, social y económica. Este atributo es generalmente de las tecnologías de gran escala y capital intensivo (como aviones y tecnologías aeroespaciales) porque no se pueden desarrollar, aplicar y organizar de una manera descentralizada y basada puramente en el mercado (Dolata, 2008). Dicho atributo lo tuvo el ferrocarril desde el siglo XIX, primer sistema sociotécnico de difusión internacional que necesitaba de cinco grandes componentes: 1) empleo de varios tipos de equipos auxiliares, estaciones, puentes, túneles, viaductos, sistemas de señalización y una enorme red de vías; 2) organización empresarial corporativa con un gran capital de inversión y mantenimiento; 3) formas especializadas de conocimientos técnicos; 4) una fuerza de trabajo calificada, que incluye ingenieros civiles y mecánicos, telegrafistas, guardafrenos, conductores y otros encargados del funcionamiento del sistema, en todo tipo de clima y durante 365 días al año, y 5) regulaciones institucionales, como el uso de indicadores estandarizados de seguimiento y de un sistema nacional de zonas normalizadas de tiempo. Estos componentes, al ser puestos en movimiento, diluían las fronteras entre el equipamiento y todo lo demás: el conocimiento técnico-científico, la fuerza de trabajo especializada, el aparato financiero y los medios para adquirir materias primas. Su complejidad y magnitud aumentó por la aplicación creciente de la ciencia a las artes mecánicas (Marx, 2010).

Mediante una organización empresarial, que desde sus inicios se ajustó al modelo de una gran empresa analizada por Chandler (1977, 1990), se gestó una complementariedad entre capitales físico y humano. Desde la segunda mitad del siglo XIX las tecnologías como el ferrocarril, el telégrafo y, posteriormente, el teléfono se organizaron en múltiples niveles, con jerarquías de conocimientos, e hicieron posible que integrara el acervo de saber científico y técnico (Goldin, 2001). Para la venta de movilidad terrestre, el ferrocarril debió integrar mercadeo, comunicación, tráfico e ingeniería al extender sus vías a través del territorio; la infraestructura fija configuró el negocio, pues funcionó de manera multilocalizada, simultánea e interactiva con su mercado mediante una organización estandarizada presente en distintos lugares con diversas instalaciones, personal, tecnologías 
e información para mover bienes y personas (Guajardo, 2009). Todas estas características lo ubicaron como una de las primeras y extensas "industrias de infraestructura", que surgieron en la década de 1830 junto con los telégrafos, las redes de gas, el agua potable, la electricidad y los tranvías, de esta manera se inicia el consumo masivo de servicios en Europa y Estados Unidos (Millward, 2005).

En América Latina y el Caribe los atributos anteriores se presentaron en casi toda su extensión y arribaron entre las décadas de 1830 y 1930 como parte de una oleada de inversiones extranjeras en empresas de ferrocarriles, electricidad, minería, fundición, refinación y transformación industrial. La transferencia tecnológica impulsó la migración y la calificación de la fuerza de trabajo local en países en donde el analfabetismo alcanzaba 80 o 90\% de la población hacia 1914 (Guajardo, 2011; Guajardo y Riguzzi, 2013). Como sistema sociotécnico abierto también influyeron factores de diverso tipo, destacando la participación del Estado; Zdeněk Tomeš (2008) indica que en el ciclo de vida del ferrocarril se ha presentado una alta dependencia de la intervención pública en su promoción, expansión y declive, situación a la cual se ajusta América Latina, donde se ha presentado una cíclica intervención del Estado.

A los atributos anteriores debemos agregar que como red de flujos se introdujo una nueva coordinación y conectividad con otros medios que intervenían en el proceso de transporte. Así ocurrió con la producción de nitrato en Chile, que se basó en una cadena en la que intervenían mulas, carretas, ferrocarriles, cargadores a hombro y balsas, caso que se tratará a continuación.

\section{LA CADENA DE MOVIMIENTO DE LA MULA AL BARCO}

Un primer caso de análisis es el de Chile, país ubicado en la costa suroeste de Sudamérica que desde el siglo XIX moldeó su sociedad y economía a través de ciclos exportadores de materias primas, predominantemente mineras: plata (1830-1860), primer ciclo del cobre (1830-1880), nitrato (1880-1930) y segundo ciclo del cobre (1915-1970); aunque en cuestiones político-sociales fue dominado por los hacendados hasta bien entrado el siglo Xx. Esta situación definió una división del trabajo entre el Estado, que asumió la construcción y operación de ferrocarriles en la zona central y sur del país en donde estaban los poderes públicos, gran parte de la población y una agricultura comercial, y el capital privado, que se encargó de construir sus ferrocarriles, caminos y puertos para cubrir las necesidades de exportación de minerales, en regiones poco habitadas y desérticas del norte del país. Esta división construyó dos subpaíses, el primero estatal y 
más integrado, el segundo mal conectado y especializado en la minería (Bulmer-Thomas, 1994; Meller, 1990).

Después de 1881, tras la anexión de los territorios de Tarapacá y Antofagasta capturados de Perú y Bolivia en la llamada Guerra del Pacífico iniciada en 1879, se expandieron la exportación de nitrato (localmente llamado salitre) y los ferrocarriles privados que atendían su transporte. Pero también las vías en manos del Estado aumentaron: de una base de 950 kilómetros, equivalentes a 53\% del país, en 1880, a 5807 kilómetros en 1930, que era 64\% de todas las vías férreas de Chile (Guajardo, 2007, p. 41). La primera guerra mundial afectó al sector exportador y retardó la inversión en los ferrocarriles privados; en cambio, las vías estatales se incrementaron entre 1910 y 1920 a casi 1700 nuevos kilómetros, frente a los 487 del sector privado (véase cuadro 1).

Si bien el Estado chileno se hizo dependiente de los ingresos de exportación del ciclo salitrero (1880-1930), la división del trabajo público-privado generó una bipolaridad, pues en el norte predominó el libre comercio y en el centro y sur del país se ejerció el intervencionismo estatal a través de las obras públicas. Esto definió un perfil específico para la producción de nitrato, fertilizante mineral que se explotaba a campo abierto en la zona desértica, al tiempo que combinaba métodos de la minería colonial con una mecanización parcial de la producción y el transporte, que conformaban una sola cadena de movimiento. Eso definió el modus tecnológico del ferrocarril, que fue operado por compañías que no estaban completamente integradas a las compañías de nitrato, porque eran un negocio independiente que muchas veces estranguló con sus tarifas al productor. En algunas zonas se ejercían conductas monopólicas, en otras se cartelizaban o bien competían por la salida a los puertos, integrándose a un flujo de operadores, hombres, mulas, carretas, lanchas, carros y locomotoras que desde los puertos en el océano Pacífico lanzaban el producto al mercado, siguiendo una orientación estricta yacimiento-puerto.

El nitrato era un producto a granel de gran volumen y peso para el cual no existían medios alternativos de transporte al puerto a través de canales y carretas, no sólo por lo desértico de la zona, sino por la gran pendiente y altura entre la pampa y la costa.

Transportar el producto con tropas de carretas se habría visto limitado por la dificultad para maniobrar y frenar la bajada, obligando a dividir una carga en varios vehículos, lo cual habría exigido construir caminos con un trazo cercano al de una línea férrea. En los puertos, la acumulación de carretas y mulas habría creado problemas, además de la escasez de conductores en una zona en donde el salitre ocupaba buena parte de la fuerza de trabajo. Por ello el transporte por caminos, desde los campos o pampas de extracción a la "oficina" o planta elaboradora, se medía en carretadas, 
CUADRO 1. CHILE: LONGITUD DE VÍAS DE FERROCARRIL DEL SECTOR PÚBLICO Y PRIVADO, 1876-1920

\begin{tabular}{lccc}
\hline Año & Kilómetros estatales & Kilómetros privados & Kilómetros totales \\
1876 & 863 & 674 & 1537 \\
1880 & 950 & 827 & 1777 \\
1890 & 1106 & 1641 & 2747 \\
1900 & 2125 & 2229 & 4354 \\
1910 & 2830 & 3144 & 5974 \\
1920 & 4579 & 3631 & 8210 \\
\hline
\end{tabular}

Fuente: Guajardo (2007, p. 43).

un método impreciso porque la capacidad de cada carreta variaba y la distancia no era tomada en cuenta. Sin embargo, ese costo era importante: si se usaban locomotoras en vez de mulas desde la extracción a la elaboración, se calculaba que la tonelada métrica costaba entre 50 y 85 centavos en 1919; en cambio, el acarreo con mulas podía llegar hasta 1.25 pesos. Tomando como base la tonelada-kilómetro, el costo medio del transporte con locomotoras a vapor habría sido de 25 centavos, y para el acarreo con mulas dos pesos. Pero en la década de 1920 se estimaba que eliminar las mulas no era fácil mientras no se adoptaran métodos más concentrados y tecnificados, a diferencia de la explotación diseminada que se practicaba. La causa de seguir utilizando mulas era que el material extraído contenía mucho nitrato y debía seleccionarse a mano, si se adoptaba un método concentrado debía explotarse un material más pobre, aunque más abundante y barato de mover con medios mecanizados (Guajardo, 2007), vía por la cual optaron las compañías estadunidenses desde fines de la década de 1920.

En ferrocarril, el costo de transportar el salitre de bajada era con un menor gasto de combustible para las locomotoras, que podía significar $12 \%$ de todo el valor del producto puesto en el barco después de los gastos de embarque y derechos de exportación. El flete era relativamente modesto respecto al valor $\mathrm{CIF},{ }^{2}$ es decir, el precio de poner la mercancía en el puerto de destino, con flete y seguro pagado. Hacia 1910, para un quintal español de salitre (46 kilogramos) el flete ferroviario entre la planta elaboradora y el puerto no representaba más de $5.5 \%$ de su valor. Pero si se mide el costo por kilómetro, el flete ferroviario era muy costoso, aunque sin constituir una fracción muy grande del precio CIF del salitre, porque la

\footnotetext{
${ }^{2}$ Acrónimo del término en inglés Cost, Insurance and Freight.
} 
distancia entre la planta elaboradora y el puerto era pocas veces superior a más de 100 kilómetros. El costo ferroviario se hacía presente al compararse con el flete marítimo; Thomson (2005) estima que antes de 1914 el flete terrestre, comparado con el flete marítimo por kilómetro entre los puertos salitreros y los europeos, como Liverpool (una distancia de casi 10000 millas náuticas), podía llegar a ser casi cien veces más caro. El transporte terrestre es normalmente más caro que el marítimo, pero lo extraordinario era la relación para el nitrato, que era de hasta 100 a uno entre el flete por kilómetro terrestre y el marítimo.

A lo anterior debe sumarse la pesadilla logística de la salida del fertilizante a los puertos de exportación mediante varias líneas férreas independientes, no todas del mismo ancho de vía, con fuertes pendientes y con una capacidad de transporte limitada en relación con el volumen total movilizado, para una carga que durante la primera guerra mundial llegó a ser de 3200000 toneladas anuales. Para la década de 1920 se calculaba que el servicio del mismo equipo rodante en un menor número de plantas elaboradoras habría podido transportar más tonelaje, pero ello habría implicado una nueva estructura económica y técnica para el salitre dada por la consolidación de los yacimientos, la centralización y la elaboración en grandes plantas. Esto habría requerido de un menor número de puertos y una red ferroviaria consolidada para bajar los costos. Pero para 1900 la red de numerosas líneas cortas tenía la ventaja de ofrecer transporte más rápido invirtiendo menos (Guajardo, 2007, pp. 161-162).

La dispersión de ferrocarriles se replicaba en la diversidad de puertos, que entregaban un volumen insuficiente de negocios para estimular la inversión privada, e incluso pública, en la construcción de rompeolas o malecones, por lo que se cargaban y descargaban los buques con lanchas y jornaleros. El fertilizante se embarcaba en sacos al hombro desde el borde de la playa hasta los botes llamados cachuchos, que recibían sólo cinco sacos. Desde los cachuchos se conducía la carga hasta las lanchas ancladas como a 40 metros de la playa, las cuales trasladaban, al fin, el nitrato al buque. Por este método los buques permanecían en promedio tres meses en la bahía de Iquique, ya que se empleaba más de un mes en la carga. Pero el problema de la mala calidad de los puertos era nacional, puesto que el carbón en el sur del país se cargaba en lanchas para llegar al costado de los vapores y desde ahí se llenaban a pala las bodegas. Una vez llegado al puerto de destino, debía descargarse nuevamente mediante una complicada cadena de brazos y hombros (Cordemoy, 1902; Guajardo, 2007, p. 162).

Esta situación empezó a cambiar lentamente desde 1911, cuando se iniciaron obras de mejoramiento en los puertos más importantes, obras que hacia 1930 estaban terminadas en Valparaíso, San Antonio, Iquique 
y Antofagasta; la explotación de los puertos chilenos hasta la década de 1930 fue realizada por agentes de las compañías de vapores o por propietarios de lanchas y muelles, quienes sólo se limitaban a construir las obras directamente remunerativas para sus intereses y no obras de abrigo y protección. A partir de esa década se incrementó el control estatal en la operación de los puertos (Lira, 1933). Junto con la mejora estuvo el lento cambio tecnológico desde la década de 1920, con el reemplazo de las carretas por camiones. Para ese entonces la mayoría de las compañías de nitrato había adoptado un método mixto en sus explotaciones mediante carretas con mulas que recorrían entre 500 y 600 metros desde los yacimientos hasta las rampas del ferrocarril, y con ferrocarriles desde las rampas a la planta elaboradora con recorridos que iban de 600 metros hasta 30 kilómetros. Pero no se podía llegar con las líneas férreas a todos los puntos de explotación por lo irregular del terreno, por lo que se seguía empleando la carreta.

A partir de 1923 se utilizaron camiones Ford con una capacidad de 1.5 toneladas; su introducción fue estimulada por la empresa constructora del puerto de Antofagasta, que reemplazó las carretas con camiones para transportar cascajo, piedras y arenas. Esta obra de infraestructura portuaria constituyó un vector tecnológico en el transporte, asociado a la transición energética del carbón al petróleo iniciado en la zona norte desde 1903 (Guajardo, 2007, pp. 162-163).

En el caso chileno los ferrocarriles fueron determinantes para hacer viable la economía exportadora y su infraestructura determinó un largo path dependence de especialización minera. Al desmenuzar las fases y medios específicos que intervenían en la cadena de movimiento salitrero, es posible encontrar que este sistema sociotécnico estableció una nueva coordinación; el ferrocarril y el telégrafo revitalizaron y proyectaron condiciones de arranque arcaicas hasta la década de 1920 que, cuando fueron cuestionadas, significaron la obsolescencia territorial masiva de la actividad de vías y puertos.

\section{INFRAESTRUCTURAS Y LOGÍSTICAS INCOMPLETAS EN EL MERCADO INTERNO}

En el caso de México, país latinoamericano ubicado en América del Norte con más de 3000 kilómetros de frontera terrestre con Estados Unidos, los viejos troncales de caminos coloniales indicaron las rutas ferroviarias que avanzaron lentamente. La primera concesión se otorgó en 1837, y la operación completa de la vía entre la ciudad de México y el puerto de Veracruz tuvo lugar en 1873. Las líneas se construyeron sobre una matriz de circulación interna establecida durante el dominio colonial que ubicó a la ciudad de México como el centro de una estrella. El plan de vías 
férreas trazado durante la ocupación francesa del país entre 1863 y 1867 pretendía complementar esa estructura con vías y caminos transversales, sin privilegiar la conexión con Estados Unidos. Pero tras la restauración republicana ello cambió, con la construcción entre 1881 y 1884, de más de 4500 kilómetros de vías que en gran parte comunicaron el centro con la frontera norte (Guajardo, 2010a).

La expansión de la nueva infraestructura se acompañó de conflictividad agraria a pesar de que los productos agrícolas mexicanos no competían en el mercado internacional, conformándose lo que Knight (1992) llamó la "clásica confrontación mexicana" (pp. 109-112), conflicto creciente entre pueblos y comunidades con los hacendados expansionistas. Lo conflictivo se incrementó con los cambios impulsados durante la dictadura de Porfirio Díaz, desde 1876 hasta 1911, cuando la combinación de inversiones en la minería de exportación en la porción norte del país, la gravitación nacional de la ciudad de México y la inversión extranjera influyeron en la construcción de ferrocarriles.

Entre 1880 y 1900 se terminaron los troncales más importantes que unieron el centro de México con tres puntos de la frontera norte: Nuevo Laredo (Nacional Mexicano), Ciudad Juárez (Central Mexicano) y Piedras Negras (Internacional Mexicano). Dos líneas comunicaban la capital con el puerto de Veracruz (Mexicano e Interoceánico) y existían vías férreas en Yucatán y el noreste. Para finales de 1910 el país contaba con poco más de 19000 kilómetros de vías férreas que consolidaron la exportación de minerales; en cambio la agricultura no recibió el mismo esfuerzo, a diferencia del caso chileno, donde el Estado proveyó infraestructura y transporte al sector agrario. Por ello México no pudo competir con el trigo argentino, con el café brasileño o con el azúcar y el tabaco de Cuba, salvo con el henequén y las fibras para el mercado estadunidense (Topik, 1994). Para 1913 México ocupaba el lugar más bajo en exportaciones transportadas por ferrocarril, gran parte de la carga consistía en piedra y materiales estériles que se movían como parte de la producción minera (véase cuadro 2).

Al respecto, Riguzzi (1994) señala que durante el periodo 1870-1911 existió la división neta del trabajo entre inversionistas extranjeros, quienes asumieron la iniciativa de expandir los ferrocarriles, e inversionistas nacionales dedicados a otras empresas. Para 1888 las compañías estadunidenses habían construido por encima de $85 \%$ de todas las líneas férreas mexicanas con inversiones por más de 80000000 de dólares, y para 1911 los capitales británico y estadunidense tenían entre 40.6 y 41.3\% de su inversión en ferrocarriles. Esta infraestructura de transportes permitió avanzar hacia otros sectores. En 1912 los empresarios estadunidenses controlaban $78 \%$ de las minas de México y $72 \%$ de todas las fundiciones, así generaron 
CUADRO 2. MÉXICO: DÓLARES ESTADUNIDENSES EXPORTADOS POR CADA KILÓMETRO DE VÍA FÉRREA, CA. 1913

\begin{tabular}{lc} 
País & Miles de dólares por kilómetro \\
& \\
Panamá & 67.1 \\
Cuba & 43.2 \\
Venezuela & 32.9 \\
Uruguay & 27.8 \\
Chile & 17.6 \\
Costa Rica & 16.9 \\
Argentina & 15.2 \\
Perú & 13.2 \\
Brasil & 12.8 \\
México & 7.2 \\
\hline
\end{tabular}

Fuente: Guajardo (2010b, p. 8).

circuitos completos de transformación. Firmas fundidoras movilizaban los minerales en bruto y refinados hacia su fundición en Monterrey y después hacia el Golfo, a la frontera norte y al puerto de Tampico. A través del Ferrocarril Internacional se conectaba con los campos carboníferos de Coahuila, en tanto que su fundición de Aguascalientes estaba vinculada a la compañía del Central. Todo esto creó una fuerte relación con la economía y población de Estados Unidos y una mayor presencia de la tecnología del ferrocarril en los estados fronterizos; en 1910, junto con las regiones del Golfo y del centro, la región norte del país tenía más de un kilómetro de vía por cada 100 kilómetros cuadrados de territorio. En relación con la población, el norte de México tenía la primacía con 28.9 kilómetros de vía por cada 10000 habitantes, en tanto que el centro del país sólo contaba con 7.7 (Guajardo, 2010b, pp. 4-6).

El crecimiento de las vías acompañó al sector exportador, en tanto que el mercado interno no dio estímulos fuertes como para hacer crecer la red ferroviaria. Gran parte de la red ferroviaria dejó fuera a vastas regiones del país, como ocurrió con las líneas de Yucatán, que para 1900 cubrían la zona productora de henequén con un total de 863 kilómetros, complementada con 576 kilómetros de líneas angostas tipo Decauville, que conectaban los campos con las estaciones. Pero muchas no tenían conexión entre ellas, eran de una baja capacidad de transporte y estaban "balcanizadas" tecnológicamente, ya que las líneas Decauville se movían con tracción animal y no eran ni una red ni un sistema. En el resto del país, desde 1880, la 
vinculación de los ferrocarriles con las haciendas en el centro del territorio nacional consolidó su viabilidad económica y amplificó sus mercados.

En la producción azucarera del estado de Morelos, hasta 1912, la modernización del transporte cañero con ferrocarriles coexistió con las Decauville arrastradas por mulas. En tanto que en la producción de cereales la ampliación de los cultivos combinó nuevas tierras con obras de irrigación limitadas, aplicación de mecanización para ciertos productos rentables y uso de animales, pero sin desplazar el empleo de arcaicos instrumentos de labranza (Guajardo, 2010b).

La falta de estímulos rurales se reflejó en el relativo empleo del ferrocarril para transportar productos agrícolas, ya que entre 1893 y 1907 dicho rubro se mantuvo relativamente estancado frente al aumento de los minerales. El escaso desarrollo de la agricultura comercial (no tropical) durante el porfiriato hizo que los cereales fueran marginales a la circulación para la exportación y el mercado interno, situación que se mantuvo hasta bien entrado el siglo XX. De ahí que algunos fenómenos de circulación verdaderamente nacionales se dieran tardíamente; recién en 1954 se enviaron 20 furgones con 1000 toneladas de trigo desde Sonora a Yucatán, esta ruta cubrió 4000 kilómetros (Guajardo, 2010b). En 1907, a pesar de que cerca de 49000000 de hectáreas de tierras fueron transferidas a particulares y compañías, no aumentó significativamente la productividad agrícola, y la mayoría de las tierras útiles estaba ocupada por haciendas y pueblos en los estados más poblados del centro; así, la producción de subsistencia cubrió estrechamente las demandas alimenticias de la población (Coatsworth, 1990, p. 175; Holden, 1994) sin dejar excedente exportable.

La revolución mexicana (1910-1920) significó un duro golpe para las infraestructuras y el ferrocarril, tanto por la destrucción y la falta de mantenimiento, como por un retraso productivo que tardó décadas en reconfigurarse. Ejemplo de ello, poco después de finalizada la revolución, fue que el hacendado azucarero Luis García Pimentel combinó tecnología del vapor con métodos agrícolas y de trabajo arcaicos para reiniciar la producción de azúcar (Womack, 1969, p. 348). Paradójicamente, las iniciativas de fomento para la modernización rural vinieron desde uno de los enemigos ideológicos de la revolución: las empresas estadunidenses.

Un primer intento se dio en 1922, cuando la International Harvester presentó al gobierno del estado de Morelos un plan para promover la venta de maquinaria agrícola, el cual requería el pago de los fletes marítimos y ferroviarios de los equipos así como del transporte de los expertos de la firma desde Nueva Orleans hasta la estación de desembarque en Morelos. A cambio, el personal de la empresa instruiría sobre el manejo de maquinaria y los nuevos métodos de trabajo, ocupando los métodos de propaganda seguidos en Estados Unidos tales como libros, folletos, ilustraciones 
gráficas y cinematográficas. Pero el gobierno de Morelos no podía pagar los fletes, por lo que solicitó ayuda al gobierno federal encabezado por Álvaro Obregón, motivo por el que la propuesta se detuvo bajo la excusa de determinar el monto del flete (Guajardo, 2010b, p. 123).

La Ford Motor tuvo más éxito. Esta firma que, a principios del decenio de 1920, promovía a escala internacional la mecanización rural, incluso en la Rusia soviética donde participaba en la tractorización de las granjas colectivas (Dalrymple, 1964). En 1925, Ford Motor solicitó al gobierno de Plutarco Elías Calles un tren para promover la venta de máquinas e implementos agrícolas, así como dar instrucción con expertos en cultivos, tractores, combustibles y refacciones. En septiembre de 1925 se concedió el tren a Ford y a 35 fábricas estadunidenses de maquinaria agrícola; el tren consistía en 20 furgones, plataformas, tanques de combustible y un coche de primera que partió desde la frontera con Estados Unidos, en Piedras Negras, estado de Coahuila, el 19 de enero de 1926, y volvió a dicha estación el 10 de abril (Guajardo, 2010b, p. 123). Durante esos meses siguió una ruta que básicamente cubrió la agricultura templada del país, sin acercarse a las zonas de cultivos tropicales como el azúcar, el café y el plátano de Morelos, Veracruz y Oaxaca. ${ }^{3}$

Es difícil medir el impacto del tren, aunque en general las zonas con más maquinaria eran aquellas cercanas a las líneas. El problema era el gran tamaño del país y la lenta velocidad de crecimiento, ya que entre 1930 y 1940 el valor real de la maquinaria agrícola en su conjunto aumentó sólo a una tasa anual promedio de $1 \%$ (Hewitt, 1988, p. 72), razón por la cual la difusión de maquinarias tuvo escasa profundidad en el campo. A principios de la década de 1930, en sus estudios sobre los datos de 3611 pueblos rurales, que contenían $17 \%$ de la población del país, Tannenbaum comprobó que 93.1\% de los pueblos no tenía acceso al ferrocarril y, por tanto, $96.5 \%$ no tenía acceso a tractores, $95.8 \%$ no estaba conectado a los telégrafos ni a información, $98.9 \%$ no tenía ingeniero y en $54.3 \%$ no había arados de acero (Wilkie, 1987, pp. 249-250, cuadro IX-3). El ferrocarril entró al siglo XX como un medio de transporte terrestre concentrado en ciertas rutas, regiones y productos, y debido a la escasez de una red de caminos, no fue desbancado radicalmente por el autotransporte de carga y pasajeros, y también porque desde la década de 1930 el cambio de medios se dio de una forma lenta y rudimentaria al protegerse el tráfico del ferrocarril, hecho que retrasó el desarrollo de los puertos y medios marítimos

\footnotetext{
${ }^{3}$ La ruta fue Saltillo-Monterrey-ciudad Victoria-Tampico-San Luis Potosí-Empalme Escobedo-Buenavista (Distrito Federal)-Hacienda de los Morales-Buenavista-Querétaro-Celaya-Acámbaro-Morelia-Ajuno-Pénjamo-Guadalajara-El Cuatro-Guadalajara-Irapuato-León-AguascalientesCañitas-Durango-Torreón-Gómez Palacio-Torreón-Hipólito-Sauceda-Monclova-Piedras Negras.
} 
hasta la década de 1970. En ese sentido, todavía falta conocer la otra cara de la distribución en el siglo Xx por el congelamiento y la coexistencia de infraestructuras y medios (véanse Guajardo, 2012; Guajardo, Salas y Velázquez, 2010).

En el caso mexicano, los ferrocarriles determinaron un largo path dependence de vinculación económica y eje de difusión de nuevas tecnologías con Estados Unidos hasta el día de hoy. Su característica fue la superposición de cadenas y la diversidad de persistencias arcaicas en un contexto geográfico en el que la minería, la agricultura y las ciudades coexistían, a diferencia de la especialización espacial de la minería chilena dada por el desierto, la baja demografía y el acceso directo al mar.

\section{CONClusiones}

Como se apuntó al inicio de este artículo, el estudio de los patrones de la trayectoria y la divergencia de una misma tecnología en distintos contextos y condiciones de arranque es una atractiva ruta de análisis histórico, que en los casos analizados arroja algunas conclusiones. Una primera es que la revolución de las infraestructuras también fue de movilidad y de coordinación de información, lo que permitió la transnacionalidad a las economías de Chile y México. Cada país definió su cadena de movimiento al combinar recursos y tecnologías de acuerdo con sus condicionamientos territoriales. Una segunda conclusión es revalorar el papel del ferrocarril no sólo como tecnología de transporte, sino como infraestructura fija que determinó un path dependence sobre el territorio y los flujos de bienes y personas que se movilizaron. Fue también una interfaz logística que introdujo mayor velocidad y una nueva coordinación de factores a través de determinados corredores.

Una tercera conclusión es que en Chile el sistema sociotécnico del ferrocarril logró ser predominante por la condición longitudinal del territorio y la división del trabajo entre inversión pública y privada, lo que definió los flujos internos y los que se daban hacia los puertos de exportación-importación. En cambio, en México la red debía atender varios polos y regiones en donde existían subsistemas de movilidad, situación distinta a la orientación este-oeste de las líneas en Estados Unidos, que en el siglo XIX siguieron y estimularon las rutas de colonización; en México ya existía un viejo asentamiento de población al arribo de las tecnologías industriales, y la red colonial de caminos sirvió de guía para los troncales ferroviarios.

Una cuarta conclusión es que Chile se vinculó más temprano al mercado internacional al conformar un sector exportador que instaló su pro- 
pia infraestructura y medios, empleó métodos coloniales de trabajo y mecanizó parte de la producción de nitrato. El ferrocarril fue relevante, pero debió coordinarse con medios no mecanizados como mulas y jornaleros, que constituyeron subsistemas sociotécnicos dispersos territorialmente por la lógica de producir sólo en yacimientos ricos. México logró una más fluida transnacionalidad en el tráfico con Estados Unidos desde la década de 1880, superando grandes distancias y dificultades territoriales, pero ello fue válido para las actividades de exportación e importación, porque en el mercado interno las redes ferroviarias no se expandieron lo suficiente por la falta de una demanda de transporte en una economía que tenía amplias islas de subsistencia, es decir, siguiendo a Wallerstein, por la existencia de segmentos no mercantilizados.

Esto último nos lleva a una quinta conclusión, que se refiere a lo señalado por O'Rourke y Williamson, en el sentido de que la magnitud del mercado de materias primas en el siglo XIX articuló un mercado global, sin embargo amplias regiones no accedieron al paquete de ferrocarriles, telégrafos e información, quedando en la condición señalada por Wallerstein. El inicio de la globalización se dio por un movimiento de coordinación de varios segmentos y unidades para convertir bienes apilables en bienes o servicios finales entregados al cliente, pero en determinados espacios y corredores territoriales.

\section{LISTA DE REFERENCIAS}

Allen, B. (1997). The logistics revolution and transportation. The annals of the American Academy of Political and Social Science, 553, 106-116.

Anastasiadou, I. (2009). In Search of a Railway Europe: Transnational railway developments in interwar Europe (Tesis doctoral). Technical University of Eindhoven, Holanda.

Baudrillard, J. (2010). El sistema de los objetos. México: Siglo XXI.

Berger, T. y Enflo, K. (julio, 2013). Locomotives of local growth: The short- and longterm impact of railroads in Sweden. European Historical Economics Society. EHES Working Papers in Economic History, 42, 1-41.

BogarT, D. (marzo, 2009). Nationalizations and the development of transport systems: Cross-country evidence from railroad networks, 1860-1912. The Journal of Economic History, 69(1), 202-237.

BogarT, D. (2013). The transport revolution in industrializing Britain: A survey. [Working Papers 121306]. University of California, Irvine, Estados Unidos.

Braudel, F. (1984). Civilización material, economía y capitalismo (3 vols). Madrid: Alianza. Bulmer-Thomas, V. (1994). The economic history of Latin America since independence. Cambridge-Nueva York: Cambridge University Press. 
Chandler JR., A. D. (1977). The visible hand: The managerial revolution in American business. Cambridge: Harvard University Press.

Chandler JR., A. D. (1990). Scale and scope. The dynamics of industrial capitalism. Cambridge: Harvard UniversityPress/Belknap.

Contsworth, J. (1990). Los orígenes del atraso. Nueve ensayos de historia económica de México en los siglos XVIII y XIX. México: Alianza Editorial Mexicana.

Cordemoy, C. J. DE (1902). Estudio relativo al puerto de Iquique. Santiago: Imprenta Cervantes.

Dalrymple, D. G. (1964). The American tractor comes to Soviet agriculture: The transfer of a technology. Technology and Culture, 5(2), 191-214

Dolata, U. (2008). The transformative capacity of new technologies. How innovations affect sectoral change: Conceptual considerations [MPIfG Discussion Paper 08/2]. Colonia, Alemania: Max Planck Institute for the Study of Societies.

Fishlow, A. (1965). American railroads and the transformation of the ante-bellum economy. Cambridge: Harvard University Press.

Fogel, R. (1964). Railroads and American economic growth: Essays in econometric history. Baltimore: The Johns Hopkins Press.

Gereffi G. y Korzeniewicz M. (eds.) (1994). Commodity chains and global capitalism. Connecticut: F. A. Praeger.

Gereffi, G., Korzeniewicz, M. y Korzeniewicz, R. (1994). Introduction: Global commodity chains. En G. GerefFi y M. Korzeniewicz (eds.), Commodity chains and global capitalism. Connecticut: F. A. Praeger.

Goldin, C. (2001). The human capital Century and American leadership: virtues of the past. NBER Working Paper 8239, National Bureau of Economic Research. Cambridge, MA, Estados Unidos.

Guajardo, G. (2001). Les modeles industriels induits par le développement du chemin de fer en Amérique latine (1850-1950). Économies et Sociétés. Série F., 35(9), 1415-1437.

Guajardo, G. (2005). La tecnología de los Estados Unidos y la 'americanización' de los ferrocarriles estatales de México y Chile, ca. 1880-1950. TST Revista de Historia de los Transportes, Servicios y Telecomunicaciones, 9, 110-130.

Guajardo, G. (2007). Tecnología, Estado y ferrocarriles en Chile, 1850-1950. Madrid y México: Fundación de los Ferrocarriles Españoles/Universidad Nacional Autónoma de México.

Guajardo, G. (2009). Between the workshop and the State: Training human capital in railroad companies in Mexico and Chile, 1850-1930. MPRA Working Paper 16135, Munich, Alemania: Personal RePEc Archive.

Guajardo, G. (2010a). Mobility history in modern Mexico: An uneven landscape. En G. Mom, P. Norton, G. Clarsen y G. Pirie (eds.), Mobility in history: Themes in transport: T2M Yearbook 2011. Neuchâtel: Éditions Alphil/Presses Universitaires Suisses.

Guajardo, G. (2010b). Trabajo y tecnología en los ferrocarriles de México: una visión histórica, 1850-1950. México: Consejo Nacional para la Cultura y las Artes. 
GujJardo, G. (2011). La calificación laboral en los ferrocarriles de Chile: la formación de grupos de eficiencia y de escuelas técnicas de empresa, 1850-1914. En I. TAUZIN-CASTELlanos (coord.), À pied, à cheval, en voiture: L'Amérique indépendante et les moyens de transport. Francia: Publications de la Maison des sciences de l'homme d'Aquitaine.

Guajardo, G. (2012). El ciclo de vida del ferrocarril y el impasse socio-técnico del transporte en México: una lectura histórica. Revista Estudios del ISHiR-CONICET, 2(3), pp. 5-16.

Guajardo, G. y Riguzzi, P. (2013). Railroad culture and mobility in twentieth Century Mexico. En A. Tinajero y J. B. Freeman (eds.), Technology and culture in Twentieth Century Mexico. Tuscaloosa: Alabama University Press.

Gujuardo, G., Salas, F. y VelázQuez, D. (2010). Energía, infraestructura y crecimiento, 1930-2008. En S. KunTz (coord.), Historia económica general de México. De la colonia a nuestros días. México: El Colegio de México/Secretaría de Economía.

Harley, C. K. (diciembre, 2000). A review of O'Rourke and Williamson's globalization and history: The evolution of a nineteenth Century Atlantic economy. Journal of Economic Literature, 38(4), 926-935.

Hesse, M. (2008). The city as a terminal: The urban context of logistics and freight transport. Burlington. Vermont: Ashgate.

HewitT, C. (1988). La modernización de la agricultura mexicana, 1940-1970. México: Siglo XXI Editores.

Holden, R. (1994). Mexico and the survey of public lands. The management of modernization, 1876-1911. Illinois: Northern Illinois University Press.

Jacks, D. S., Meissner, C. M. y Novy, D. (2010). Trade costs in the first wave of globalization. Explorations in Economic History, 47(2), 127-141.

Kaufmann, V. (2010). Mobility: Trajectory of a concept in the social sciences. En G. Mom, G. Pirie y L. Tissot (eds.), T2M Yearbook 2009. Mobility in history. The state of the art in the history of transport, traffic and mobility. Neuchâtel: Éditions AlphilPresses Universitaires Suisses

Knight, A. (marzo, 1992). The peculiarities of Mexican history: Mexico compared to Latin America, 1821-1992. Journal of Latin American Studies, Quincentenary Supplement, 24, 99-144.

LIRA, J. (1933). Puertos chilenos. Santiago: Imprenta Nascimento.

MARX, L. (2010). Technology: The emergence of a hazardous concept. Technology and Culture, 51(3), 561-577.

Meller, P. (1990). Una perspectiva de largo plazo del desarrollo económico chileno, 1880-1990. En M. Blomström y P. Meller (eds.), Trayectorias divergentes. Comparación de un siglo de desarrollo económico latinoamericano y escandinavo. Santiago: Corporación de Estudios para Latinoamérica [CIEPLAN]/Hachette.

MeYer, J. R. (septiembre, 1997). Transportation today: The U.S. experience in a world context. The Annals of the American Academy of Political and Social Science, 553(1), pp. 17-29. 
Millward, R. (2005). Private and public enterprise in Europe. Energy, telecommunications and transport, 1830-1990. Cambridge: Cambridge University Press.

Mom, G., Divall, C. y LyTh, P. (2009). Towards a paradigm shift? A decade of transport and mobility history. En G. Mom, G. PIRIE y L. Tissot (eds.), T2M Yearbook 2009. Mobility in history. The state of the art in the history of transport, traffic and mobility. Neuchâtel: Éditions Alphil-Presses Universitaires Suisses.

O'Rourke, K. H. y Williamson, J. G. (1999). Globalization and history: The evolution of a nineteenth Century Atlantic economy. Cambridge: MIT Press.

O'Rourke, K. H. y Williamson, J. G. (2000). When did globalization begin? NBER Working Paper 7632. National Bureau of Economic Research. Cambridge, Massachusetts, Estados Unidos.

O'Rourke, K. H. y Williamson, J. G. (2004). Once more: When did globalization begin? European Review of Economic History, 8, 109-117.

Riguzzi, P. (1994). "Foreign Financing of Mexican Railways, 1880-1914: Patterns and Impact on the Economic Space". Ponencia presentada al XI International Economic History Congress, Milán, Italia.

Thomson, I. (2005). La Nitrate Railways Co. Ltd.: La pérdida de sus derechos exclusivos en el mercado del transporte de salitre y su respuesta a ella. Historia, 38(1), 85-112.

TOMEš, Z. (2008). Applying the life-cycle theory: the rise and fall of railways. Journal of Transport History, 29(1), 120-124.

TopIK, S. C. (octubre de 1994). The image of prosperity: The Porfirian export boom in comparative perspective. Presentado en XI Congreso de Historiadores Mexicanos, Canadienses y Estadunidenses, ciudad de México.

VAHRENKAMP, R. (2012). The logistic revolution: The rise of logistics in the mass consumption society. Lohmar: Josef Eul Verlag.

VAn Der Vleuten, E. (2008). Toward a transnational history of technology: Meanings, promises, pitfalls. Technology and Culture, 49(4), 974-994.

Wallerstein, I. (2010). El capitalismo histórico (1a. ed. inglés 1983). México: Siglo XXI.

Wenzlhuemer, R. (2010). Globalization, communication and the concept of space in global history. Historical Social Research, 35(1), 19-47.

WiLKIE, J. W. (1987). La revolución mexicana (1910-1976). Gasto federal y cambio social. México: Fondo de Cultura Económica.

Womack, J. (1969). Zapata y la revolución mexicana. México: Siglo XXI Editores. 